\title{
Role of dynamin, synaptojanin, and endophilin in podocyte foot processes
}

\author{
Keita Soda, ${ }^{1}$ Daniel M. Balkin, 2,3,4 Shawn M. Ferguson,, ${ }^{2,4}$ Summer Paradise, ${ }^{2,3,4}$ Ira Milosevic, ${ }^{2,3,4}$ \\ Silvia Giovedi, ${ }^{2,3,4}$ Laura Volpicelli-Daley, ${ }^{2,3,4}$ Xuefei Tian, ${ }^{1}$ Yumei Wu, ${ }^{2,3,4}$ Hong Ma, ${ }^{1}$ \\ Sung Hyun Son, ${ }^{1}$ Rena Zheng, ${ }^{1}$ Gilbert Moeckel, ${ }^{5}$ Ottavio Cremona, ${ }^{6}$ \\ Lawrence B. Holzman,7 Pietro De Camilli,2,3,4 and Shuta Ishibe'
}

${ }^{1}$ Department of Internal Medicine, ${ }^{2}$ Department of Cell Biology, ${ }^{3}$ Howard Hughes Medical Institute,

${ }^{4}$ Program In Cellular Neuroscience, Neurodegeneration, and Repair (CNNR), and 5 Department of Pathology, Yale University School of Medicine,

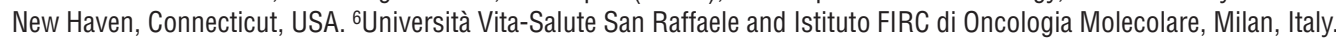
7Department of Medicine, University of Pennsylvania, Philadelphia, Pennsylvania, USA.

\begin{abstract}
Podocytes are specialized cells that play an integral role in the renal glomerular filtration barrier via their foot processes. The foot processes form a highly organized structure, the disruption of which causes nephrotic syndrome. Interestingly, several similarities have been observed between mechanisms that govern podocyte organization and mechanisms that mediate neuronal synapse development. Dynamin, synaptojanin, and endophilin are functional partners in synaptic vesicle recycling via interconnected actions in clathrin-mediated endocytosis and actin dynamics in neurons. A role of dynamin in the maintenance of the kidney filtration barrier via an action on the actin cytoskeleton of podocytes was suggested. Here we used a conditional double-KO of dynamin $1(D n m 1)$ and $D n m 2$ in mouse podocytes to confirm dynamin's role in podocyte foot process maintenance. In addition, we demonstrated that while synaptojanin 1 (Synj1) KO mice and endophilin 1 (Sh3gl2), endophilin 2 (Sh3gl1), and endophilin 3 (Sh3gl3) triple-KO mice had grossly normal embryonic development, these mutants failed to establish a normal filtration barrier and exhibited severe proteinuria due to abnormal podocyte foot process formation. These results strongly implicate a protein network that functions at the interface between endocytosis and actin at neuronal synapses in the formation and maintenance of the kidney glomerular filtration barrier.
\end{abstract}

\section{Introduction}

Nephrotic syndrome is a severe kidney disease involving massive protein loss in the urine due to an impairment of the glomerular filtration barrier between the blood and the urinary space. Podocytes, highly specialized cells that line the capillary loops, play a key role in this barrier via their interdigitated foot processes connected by a thin diaphragm, the slit diaphragm (1). Accordingly, disruption of foot process organization (a process called effacement) results in nephrotic syndrome (2). Several similarities have emerged between protein networks underlying the development and maintenance of podocyte foot processes and those governing the development and function of neuronal synapses $(3,4)$.

A study based on cathepsin-dependent proteolysis or expression of a dominant-negative dynamin mutant in podocytes provided evidence supporting a role of dynamin in foot process maintenance (5). Dynamin, which is encoded by 3 genes in mammals (dynamin 1 [Dnm1] and Dnm3, which are expressed primarily in brain, and $D n m 2$, which is ubiquitously expressed), is a GTPase that mediates the fission reaction of clathrin-mediated endocytosis $(6,7)$. At neuronal synapses, where dynamin is represented primarily by Dnm1, this protein plays a critical role in synaptic vesicle endocytosis and recycling (8-10). Dynamin has also been implicated in actin dynamics because of its colocalization with Arp2/3-dependent actin structures and its direct interaction with actin and with actin-regulatory proteins (11-13). Genetic studies in mice and humans have demonstrated the critical dependence of podocyte

Authorship note: Keita Soda and Daniel M. Balkin contributed equally to this work. Conflict of interest: The authors have declared that no conflict of interest exists. Citation for this article: J Clin Invest. 2012;122(12):4401-4411. doi:10.1172/JCI65289. foot process integrity, and thus of kidney filtration barrier function, on actin-regulatory proteins and upstream signaling factors, consistent with actin being a core component for normal podocyte architecture (14). Thus, it has been proposed that the function of dynamin in podocytes may be mediated by its role in the actin cytoskeleton $(5,11)$. However, clathrin-coated pits and vesicles are frequently observed in podocyte foot processes (15), raising the possibility that endocytic defects may contribute to foot process abnormalities upon disruption of dynamin's function.

In this study, we first addressed the role of dynamin in the stability of podocyte foot processes using a conditional gene KO strategy, and demonstrated that podocyte-selective KO of $D n m 1$ and $D n m 2$ resulted in severe proteinuria, renal failure, and foot process effacement. Next, we investigated whether 2 major functional partners of dynamin at neuronal synapses, synaptojanin (16-18) and endophilin $(19,20)$, are also implicated in the formation and maintenance of the kidney glomerular filtration barrier. Synaptojanin, an inositol 5-phosphatase whose preferred substrates are phosphatidylinositol 4,5-bisphosphate (PIP2) and phosphatidylinositol 3,4,5-trisphosphate (PIP3), plays a critical role in clathrin-coated dynamics and in the uncoating of newly formed clathrin-coated vesicles (17). It is also a negative regulator of actin nucleation and signaling (21, 22). Endophilin is an adaptor that binds the membrane bilayer via its N-terminal region (which contains a BAR domain and amphipathic helices) and both dynamin and synaptojanin via its C-terminal SH3 domain, thus helping coordinate the function of these proteins in endocytosis $(19,20,23)$. Analysis of mice harboring germline $\mathrm{KO}$ mutations of these proteins showed that while the lack of synaptojanin 1 (Synj1) or of all 3 endophilins 
A
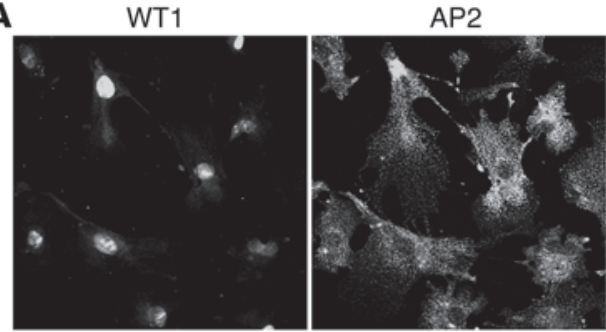

C
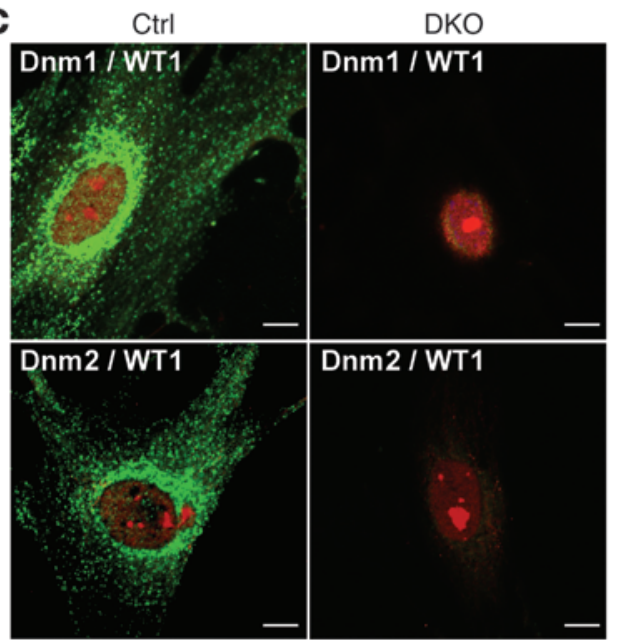

$\mathbf{F}$
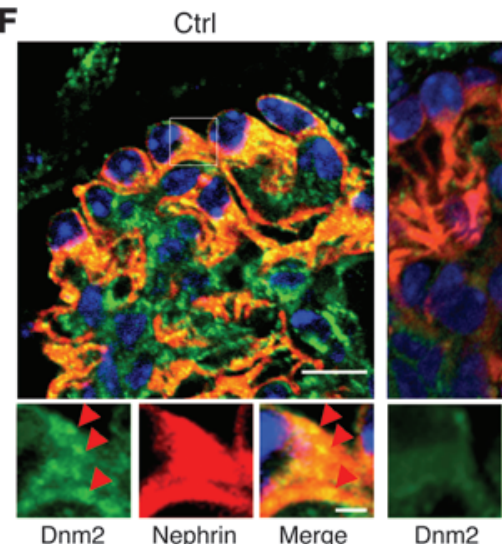

DKO

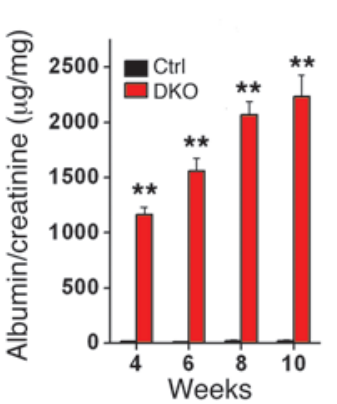

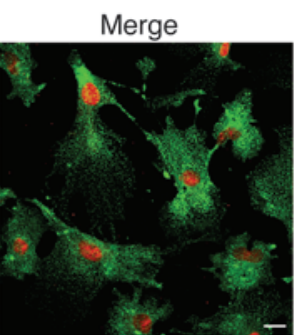

D

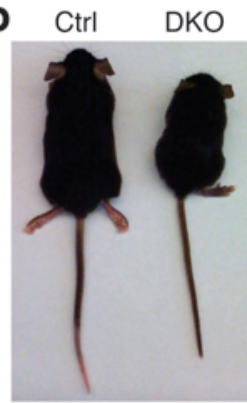

B

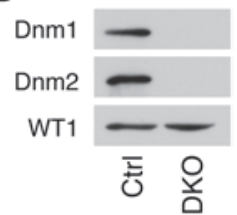

E

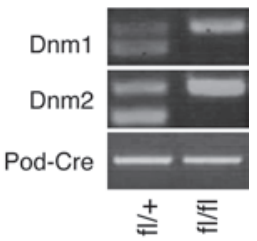

\section{Figure 1}

Loss of Dnm1 and Dnm2 in podocytes results in proteinuria and renal failure. (A) Cultured purified podocytes immunostained for the podocytespecific nuclear marker WT1 and the endocytic clathrin adaptor AP2. Scale bar: $30 \mu \mathrm{m}$. (B and $\mathbf{C}$ ) Expression of both Dnm1 and Dnm2 in purified control podocytes, identified by WT1 staining, and loss of both proteins in pod-Dnm-DKO podocytes (age P21), as detected by Western blotting and immunofluorescence. Scale bars: $10 \mu \mathrm{m}$. (D) Control and pod-Dnm-DKO mice at 10 weeks of age. (E) Dnm1, Dnm2, and Podocin genotypes, as confirmed by tail genotyping in podDnm-DKO mice (age P10). (F) Double immunofluorescence detection of nephrin (red) and Dnm2 (green) on kidney sections of the indicated genotypes (age P21). Scale bars: $10 \mu \mathrm{m}$ (top); $2 \mu \mathrm{m}$ (bottom). (G) SDS-PAGE (Coomassie Blue staining) of $0.5,1$, or $10 \mu \mathrm{g}$ BSA standard and of urine from pod-Dnm-DKO mice, which demonstrated albuminuria at 4 and 8 weeks. $2 \mu \mathrm{l}$ urine was loaded per lane. $(\mathbf{H})$ Quantification of urinary albumin normalized to creatinine at $4,6,8$, and 10 weeks $(n=6)$. (I) Elevated plasma creatinine in pod-Dnm-DKO mice at 4,8 , and 10 weeks of age $(n=6)$. ${ }^{\star} P<0.005,{ }^{* \star} P<0.001$ vs. control.

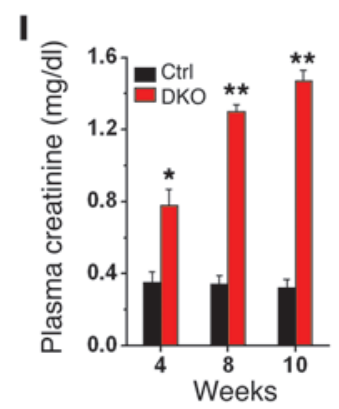

did not produce macroscopic defects in embryonic development, the glomerular filtration barrier was not properly developed at birth in either genotype, resulting in massive proteinuria in newborn mice. We further determined that, as at neuronal synapses, all these proteins functioned at sites of clathrin-mediated endocytosis in podocytes. Interestingly, dynamin, synaptojanin, and endophilin bound either directly or indirectly to proteins that genetic studies have implicated in human nephrotic syndrome. Our results indicate that a protein network that operates at the interface between endocytosis and actin at neuronal synapses is additionally critical in the formation and maintenance of the kidney glomerular filtration barrier. 
A
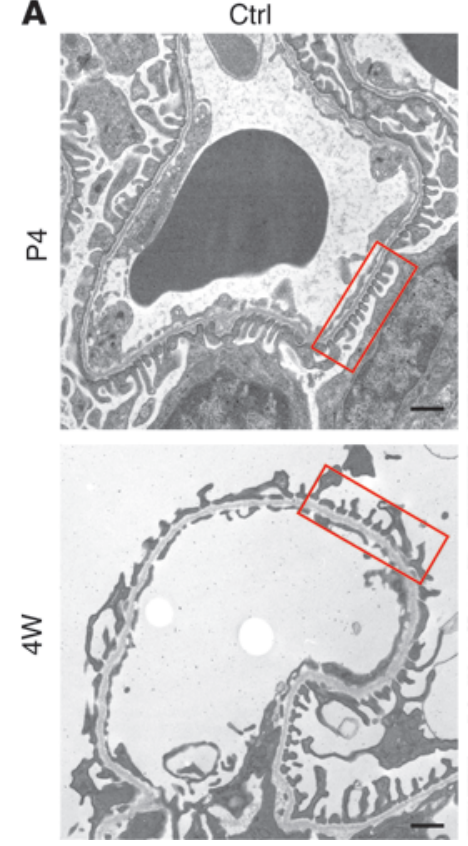
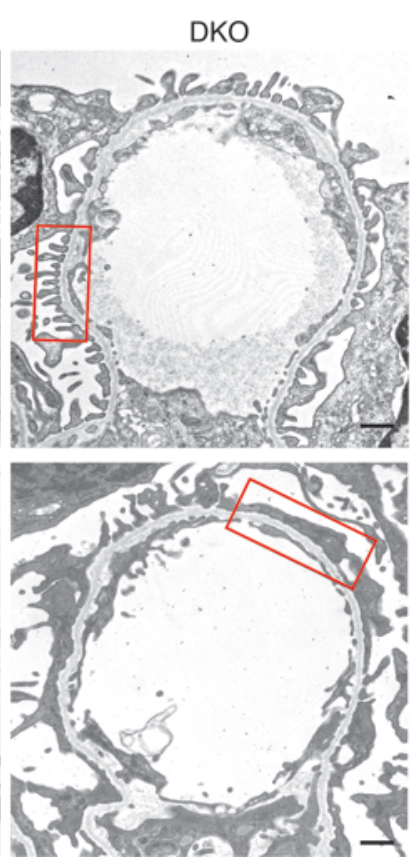

B

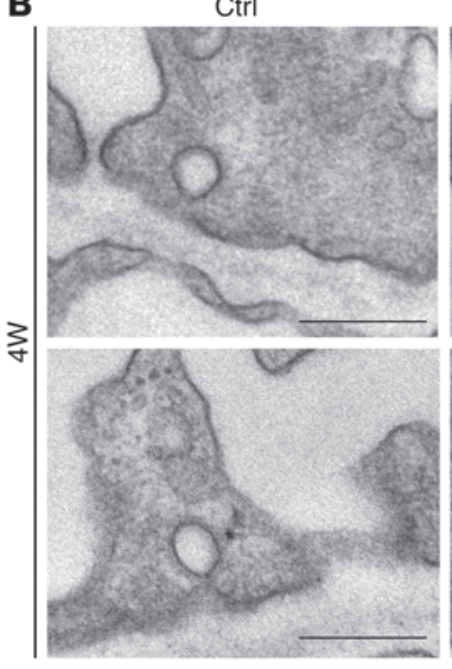

DKO

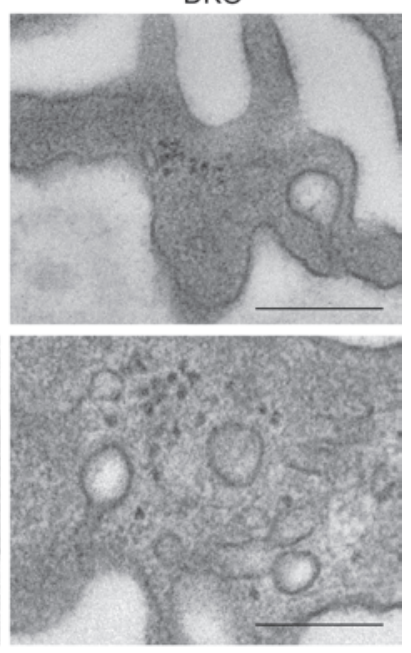

Figure 2

Podocyte foot process effacement in pod-Dnm-DKO mice. (A) Electron micrographs illustrating the ultrastructure of glomerular capillaries of control and pod-Dnm-DKO mice. In pod-Dnm-DKO capillaries, podocyte foot processes had a nearly normal morphology at P4, but were effaced at 4 weeks. Scale bars: $500 \mathrm{~nm}$. Boxed regions illustrate effaced pod-Dnm-DKO podocyte foot processes at 4 weeks compared with control. (B) High-magnification micrographs from control and mutant glomeruli showing endocytic clathrin-coated pits at 4 weeks of age. Scale bars: $500 \mathrm{~nm}$.

\section{Results}

Loss of Dnm1 and Dnm2 in podocytes results in proteinuria and kidney failure. We first revisited the role of dynamin in the maintenance of the kidney glomerular filtration barrier using a $\mathrm{KO}$ approach. Podocytes were expected to selectively express Dnm2, the housekeeping and ubiquitous dynamin isoform, whose constitutive $\mathrm{KO}$ results in embryonic lethality (6). Surprisingly, however, immunoisolated podocytes (24), as validated by their reactivity for the podocyte-specific marker WT1 (Figure 1A), were found to express not only Dnm2, but also Dnm1 (Figure 1, B and C), the predominantly neuronal dynamin (9). Thus, we used previously described $D n m 1^{f l / f l}$; $D n m 2^{f l f l}$ mice (6) and mice expressing Cre recombinase under the control of the podocin promoter (Cre expression occurs at E14; ref. 25 ) to generate podocyte-specific Dnm1;Dnm2 double-KO (referred to herein as pod-Dnm-DKO) mice. Such mice appeared normal at birth, but their weight gain fell below that of $D n m 1^{f / f l} ; D n m 2^{f / f l}$ control mice in the following days (Supplemental Figure 1B; supplemental material available online with this article; doi:10.1172/ JCI65289DS1). By 10 weeks of age, they were severely lethargic and much smaller than controls (Figure 1D). More than $75 \%$ of podDnm-DKO mice died between 10 and 12 weeks of age.

PCR-based tail genotyping confirmed the presence of the conditional dynamin alleles as well as the podocin Cre transgene (Figure 1E), and Western blot analysis of podocytes purified from pod-Dnm-DKO mice revealed that Dnm1 and Dnm2 levels were already somewhat reduced at E16.5 relative to control littermates (Figure 1, B and C, and Supplemental Figure 1A). Gene ablation of Dnm1 and Dnm2 expression was further confirmed by immunofluorescence of purified pod-Dnm-DKO podocytes extracted from P21 mice (Figure 1C) and of P21 pod-Dnm-DKO kidney sections counterstained with the podocyte-specific protein nephrin (Figure 1F). Importantly, robust albuminuria was detected in the urine of pod-Dnm-DKO mice, as assessed by SDSPAGE at 4 and 8 weeks (Figure 1G) and quantified by ELISA with normalization to urine creatinine at several ages (Figure $1 \mathrm{H}$ and Supplemental Figure 2A). Moreover, measurement of plasma creatinine levels in pod-Dnm-DKO mice demonstrated severe kidney failure (Figure 1I and Supplemental Figure 2B).

The kidney/body weight ratio in pod-Dnm-DKO mice was significantly reduced at 8 weeks of age (50\% reduction; Supplemental Figure 1C), and the kidneys displayed a pale, smaller, and corrugated appearance (Supplemental Figure 1D). While the kidneys were normal at birth, mesangial expansion and evidence of focal segmental glomerulosclerosis was observed by 4 weeks of age (Supplemental Figure 1E). By 10 weeks of age, the glomeruli had undergone a process of global sclerosis, and the kidney further demonstrated severe interstitial fibrosis and tubular dilatation with proteinaceous casts (Supplemental Figure 1F). This time course, with normal development but then loss of foot processes, is consistent with induced rather than constitutive loss of dynamin and with the use of a transgenic mouse in which Cre expression requires podocyte differentiation.

Correspondingly, the glomeruli of kidneys from neonatal podDnm-DKO mice appeared relatively normal by electron microscopy, although heterogeneity was observed, with the occurrence of some effaced capillary loops (Figure 2A and Supplemental Figure $3 \mathrm{~A})$. However, at 4 weeks of age, kidneys from pod-Dnm-DKO mice revealed generalized podocyte foot process effacement (Figure 2, A and B), which became even more severe at 8 weeks (data not shown). Additionally, clathrin-coated pit intermediates were fre- 
A

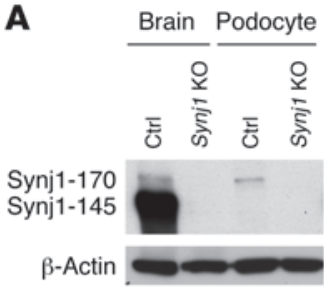

$\mathbf{F}$

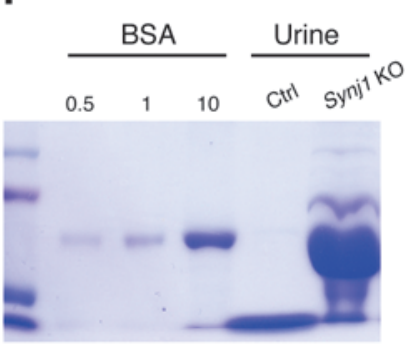

B
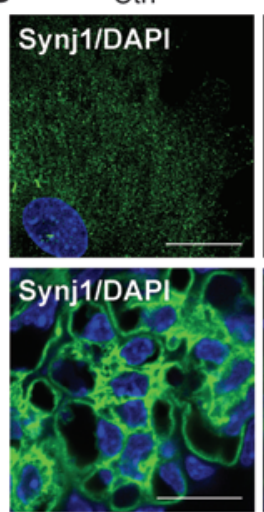

Synj1 KO
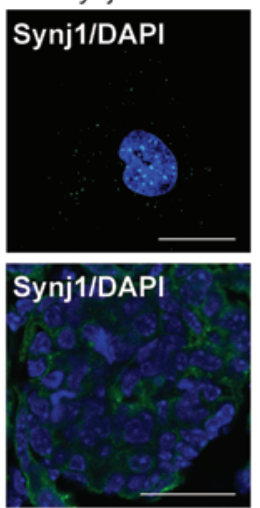

C
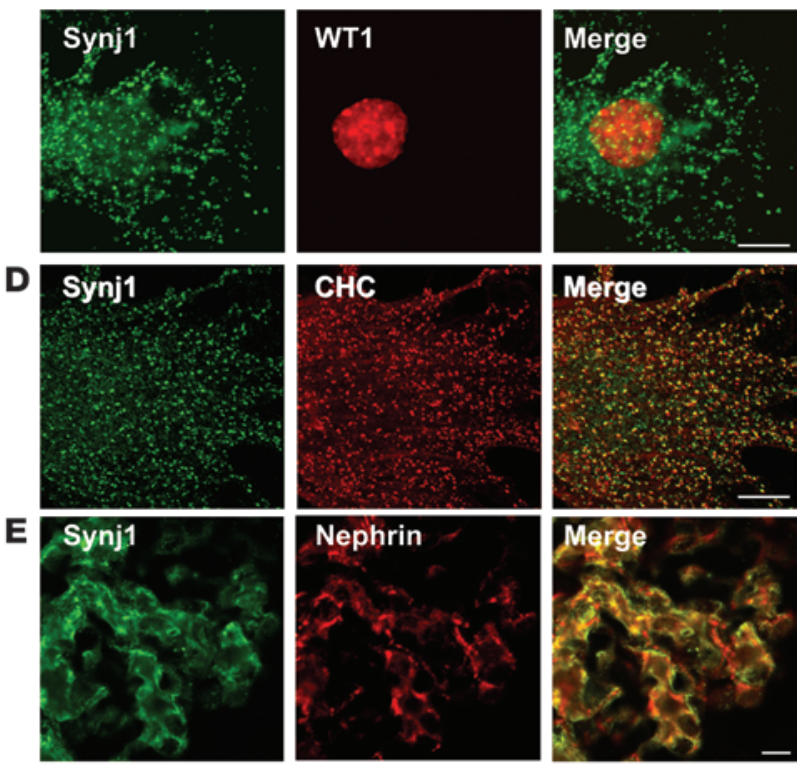

G

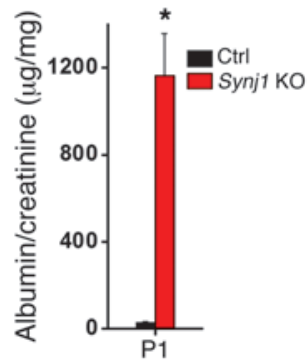

H

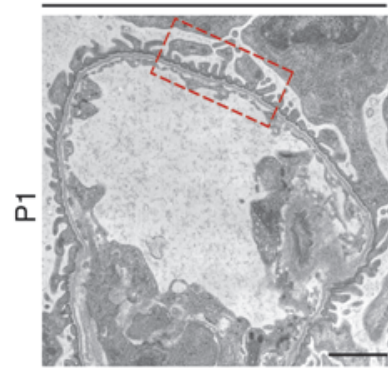

Synj1 KO

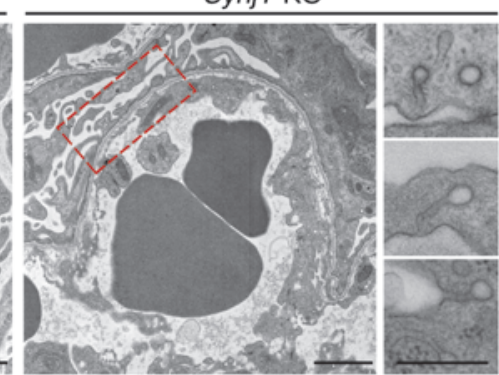

Figure 3

Lack of Synj1 results in severe proteinuria and foot process effacement. (A) Western blots of total homogenates of brain and purified podocytes of neonatal control and Synj1 KO mice, demonstrating that podocytes selectively expressed Synj1-170, not Synj1-145. $\beta$-Actin served as a loading control. (B) Synj1 immunofluorescence staining of isolated primary podocytes and kidney sections of control and Synj1 KO mice. Scale bars: $10 \mu \mathrm{m}$ (top); $30 \mu \mathrm{m}$ (bottom). (C) Immunofluorescence for Synj1 and for the podocyte nuclear marker WT1 on an isolated podocyte. Scale bar: $20 \mu \mathrm{m}$. (D) Colocalization of Synj1 and clathrin heavy chain (CHC) in cultured podocytes. Scale bar: $20 \mu \mathrm{m}$. (E) Colocalization of Synj1 and nephrin in the glomerulus. Scale bar: $20 \mu \mathrm{m}$. (F) SDS-PAGE (Coomassie Blue stain) of 0.5, 1, or $10 \mu \mathrm{g}$ BSA standard and of urine (2 $\mu$ ) from control and Synj1 KO neonatal mice. (G) Quantification of urinary albumin normalized to creatinine $(n=12)$. ${ }^{*} P<0.001$ vs. control. (H) Electron micrographs from glomeruli of control and Synj1 KO neonatal mice revealing foot process effacement and clathrin-coated intermediates in Synj1 $\mathrm{KO}$ mice. Boxed regions illustrate effaced podocytes in Synj1 KO mice compared with control. Scale bars: $500 \mathrm{~nm}$.

quently observed in such processes (Figure 2B), consistent with the occurrence of clathrin-mediated endocytosis in wild-type podocyte foot processes (Figure 2B, Supplemental Figure 3B, and ref. 15).

Proteinuria and podocyte foot process effacement in Synj $1 \mathrm{KO}$ mice. The severe effect on glomerular filtration produced by the lack of dynamin selectively in podocytes could in principle be attributed to a critical role of dynamin for the function of any cell in which it is expressed, although cells remain viable in the absence of dynamin (6). Thus, we examined the effect on kidney physiology of Synj1, which functions in close partnership with dynamin at synapses (17, 18, 26), but does not have critical housekeeping functions. Synj1 KO mice appear grossly normal at birth, although they are neurologically impaired and die within the first few postnatal days (17). Synj1 is a phosphoinositide phosphatase that dephosphorylates PIP2 and PIP3, 2 phosphoinositides that help recruit endocytic clathrin coats to the plasma membrane and whose dephosphorylation is needed for clathrin uncoating after fission $(18,22)$. The Synj1 gene encodes 2 major splice variants. The $170-\mathrm{kDa}$ splice variant Synj1-170, which has a broad tissue distribution (27), contains C-terminal binding sites for clathrin as well as other endocytic adaptors, is recruited early to endocyte-coated pits, and remains associated with them throughout their growth (28). The $145-\mathrm{kDa}$ synaptic variant Synj1145 , which is selectively expressed in the developed nervous tissue and is present at very high concentrations at neuronal synapses, is recruited only to late-stage endocytic clathrin-coated pits (28). Western blotting of isolated podocytes revealed the presence of Synj1, specifically the Synj1-170 isoform (Figure 3A). Correspondingly, Synj1 immunofluorescence demonstrated the punctate pattern of immunoreactivity expected for the clathrin-coated pit localization of this isoform, whereas such reactivity was lost in the podocytes and kidneys of Synj1 KO mice (Figure 3, B-D). Moreover, Synj1 colocalized with nephrin in podocytes within the glomerulus (Figure 3E). 


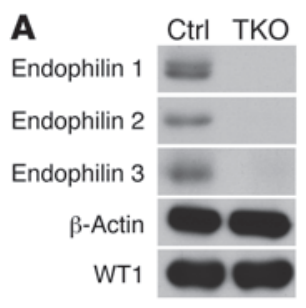

B
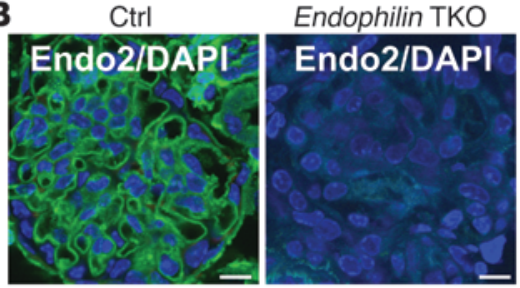

D
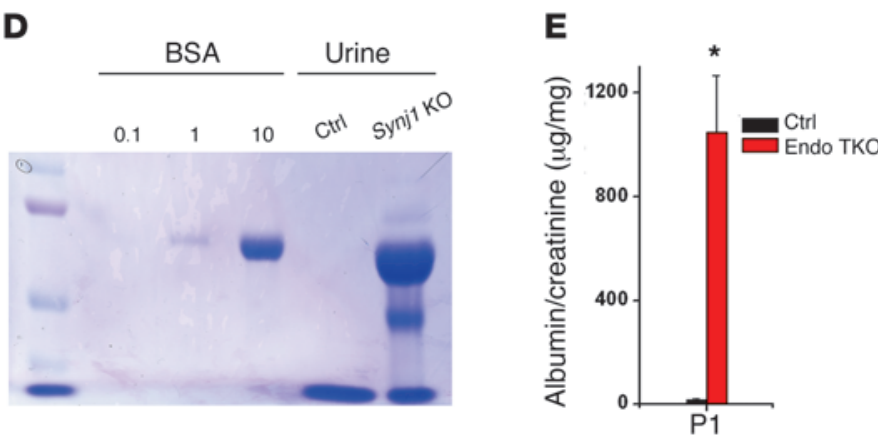

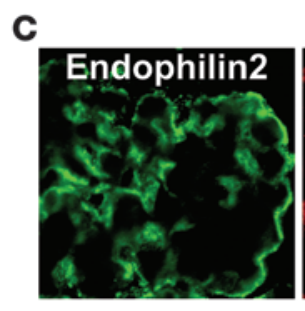

$\mathbf{F}$

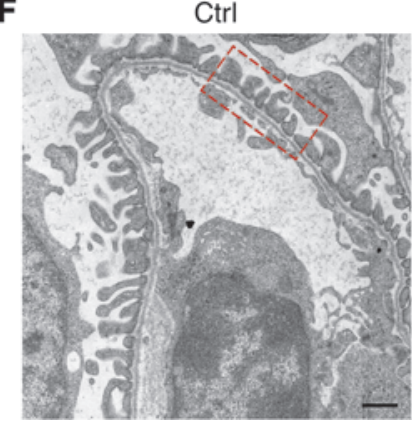

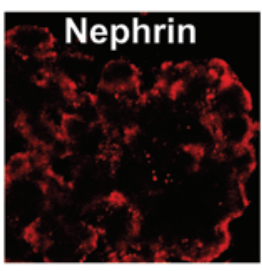

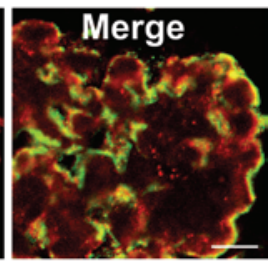

Endophilin TKO

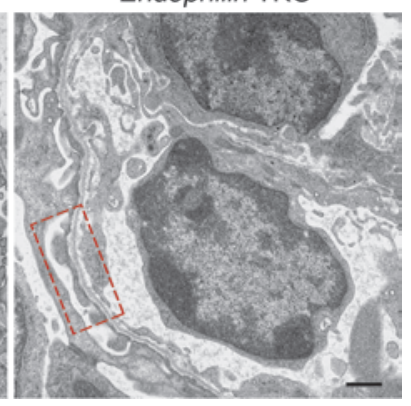

\section{Figure 4}

Lack of endophilin results in severe proteinuria and foot process effacement. (A) Western blots of purified podocytes of neonatal control and endophilin TKO mice (P0) demonstrating loss of endophilin 1, 2, and 3 in endophilin TKO mice. WT1 staining was used as a loading control. (B) Endophilin 2 immnuofluorescence staining of glomeruli within kidney sections of control and endophilin TKO mice. Scale bar: $10 \mu \mathrm{m}$. (C) Colocalization of endophilin 2 with nephrin in the glomerulus. Scale bar: $10 \mu \mathrm{m}$. (D) SDS-PAGE (Coomassie Blue staining) of $0.5,1$, or $10 \mu \mathrm{g}$ BSA standard and of urine $(2 \mu \mathrm{l})$ from control and endophilin TKO neonatal mice. (E) Quantification of urinary albumin normalized to creatinine $(n=7)$. ${ }^{\star} P<0.001$ vs. control. (F) Electron micrographs from glomeruli of control and endophilin TKO neonatal mice revealing foot process effacement. Boxed regions illustrate effaced podocytes in endophilin TKO mice compared with control. Scale bars: $500 \mathrm{~nm}$.

We next analyzed the effect of the lack of Synj1 on kidney function in Synj1 KO mice. Histological examination of neonatal kidneys revealed that some glomeruli appeared to have mesangial matrix accumulation (Supplemental Figure 4A). Dilated tubules with proteinaceous casts were also present (Supplemental Figure 4B), suggestive of severe proteinuria. Moreover, PIP2 levels, as detected by an HPLC-based method, were $25 \%$ higher in kidneys of newborn $\mathrm{KO}$ mice relative to kidneys of control littermates (Supplemental Figure 4C), demonstrating an important effect of Synj1 on PIP2 metabolism in this tissue.

Importantly, urine analysis of P1-P4 Synj1 KO mice revealed prominent albuminuria (Figure $3 \mathrm{~F}$ ) that was confirmed by normalization to urine creatinine (Figure 3G and Supplemental Figure 2C), consistent with disruption of the glomerular filtration barrier. Accordingly, electron microscopy of kidneys of P1-P4 Synj1 KO mice revealed massive foot process effacement (Figure $3 \mathrm{H}$ and Supplemental Figure $3 \mathrm{~A}$ ). As in pod-Dnm-DKO mice, the podocytes of Synj1 KO mice also exhibited numerous clathrincoated endocytic intermediates. Significant foot process effacement was already observed in E16.5 embryos (Supplemental Figure 4D), which indicates that Synj1 is involved not only in the maintenance, but also in the development, of the filtration barrier.

Proteinuria and podocyte foot process effacement in endophilin triple-KO (TKO) mice. In the nervous system, the effect of the lack of Synj1 (primarily clathrin uncoating) is strikingly phenocopied by the lack of endophilin, which indicates that these 2 proteins work closely together $(20,29-31)$. Endophilin is the collective name for 3 very similar proteins, endophilin 1, 2, and 3 (encoded by $S h 3 g l 2$, $S h 3 g l 1$, and $S h 3 g l 3$, respectively). Endophilin is recruited to the necks of endocytic clathrin-coated pits by its curvature sensitive
BAR domain $(32,33)$ and binds both dynamin and synaptojanin (with 10-fold higher affinity for synaptojanin than for dynamin; refs. 19, 23) via its C-terminal SH3 domain, thus helping recruit these proteins to their sites of action. Thus, it was of interest to determine whether the lack of endophilin phenocopies the lack of Synj 1 in the organization of the glomerular filtration barrier.

Like Synj1 KO mice, endophilin TKO mice (with triple-KO of $S h 3 g l 2$, Sh3gl1, and Sh3gl3) have grossly normal embryonic development, which argues against essential housekeeping functions of endophilin. Like Synj1 KO mice, they die perinatally, at least in part because of impaired neuronal function. Western blot analysis of isolated podocytes demonstrated expression of all 3 endophilins that was lost in the endophilin TKO, as expected (Figure 4A). Immunofluorescence staining of kidney sections from wild-type newborn mice revealed endophilin 2 expression in control, but not endophilin TKO, podocytes, which were identified by the presence of immunoreactivity for nephrin (Figure 4, B and C). As observed in Synj1 KO mice, histological examination of the endophilin TKO kidney cortex showed abnormalities in the glomeruli and dilated tubules with proteinaceous casts (Supplemental Figure 5, A and B). Urine obtained from these mice revealed robust proteinuria, as assessed by Coomassie Blue staining and further validated by normalization to urine creatinine (Figure 4, D and E, and Supplemental Figure 2D). Importantly, severe foot process effacement with a thickened glomerular basement membrane was observed by electron microscopy at birth (Figure 4F and Supplemental Figure 3A), as in Synj1 KO mice.

Clathrin-mediated endocytosis and actin in podocytes. Collectively, the findings described here suggest that the functional partnership among dynamin, synaptojanin, and endophilin at endocytic sites, 
A

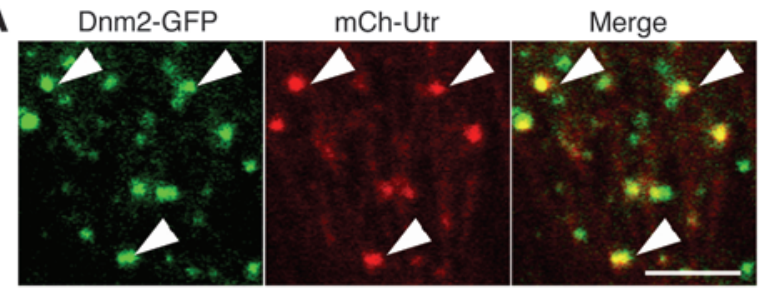

C

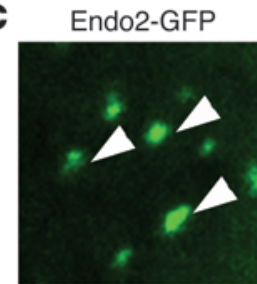

$\mathrm{mCh}-\mathrm{Utr}$

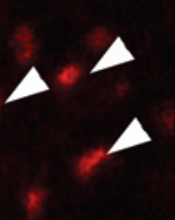

E

E Ctrl

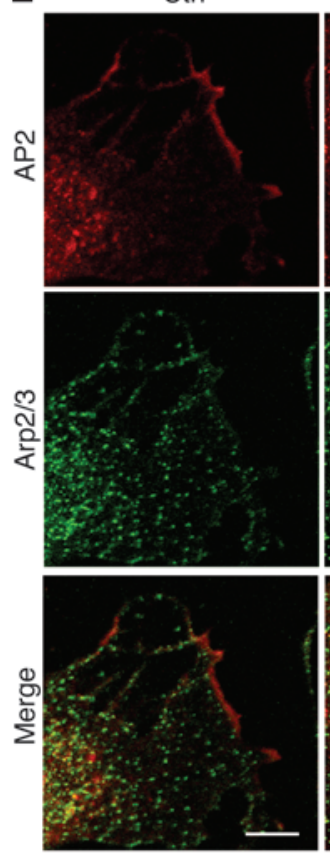

I

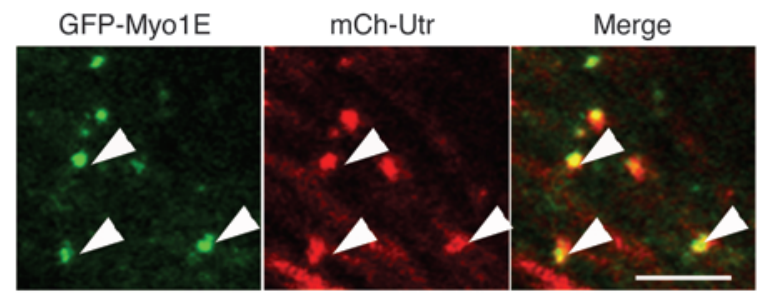

$\mathbf{K}$

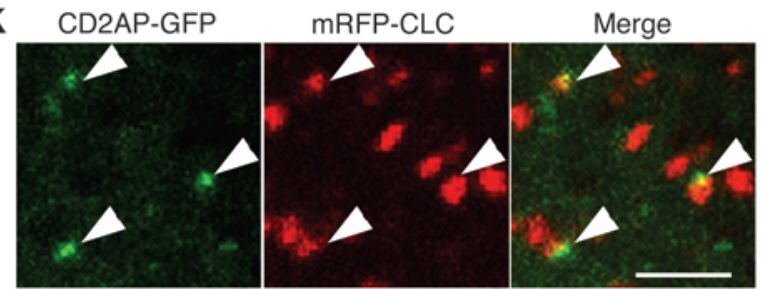

B

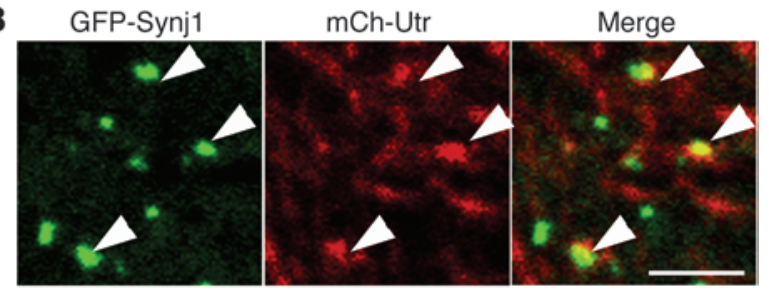

D

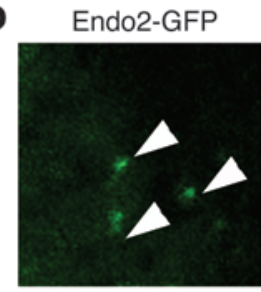

$\mathbf{F}$
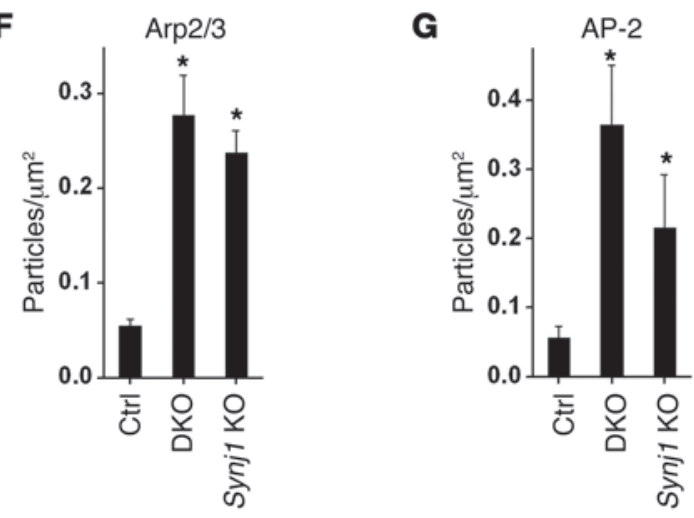

H Endophilin
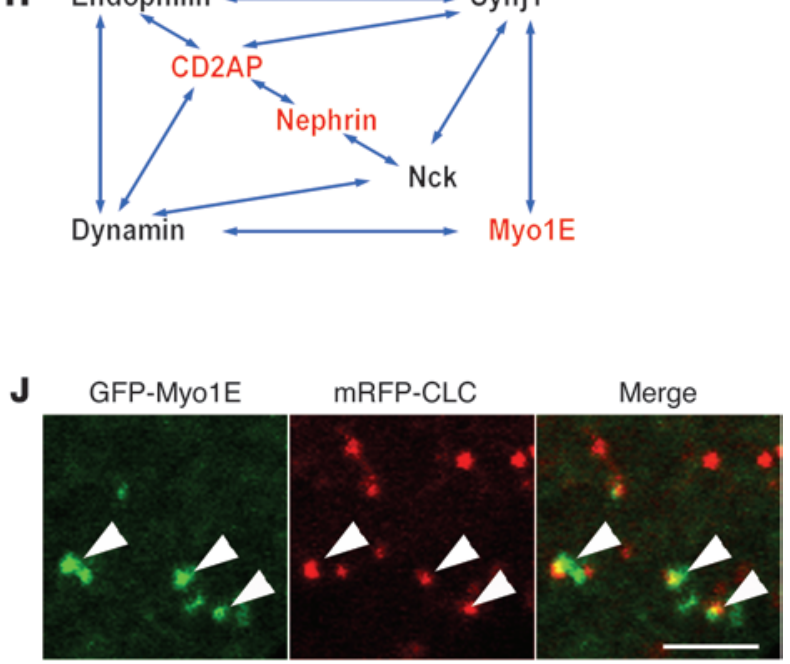


\section{Figure 5}

Defects in actin regulation and endocytosis in pod-Dnm-DKO, Synj1 $\mathrm{KO}$, and endophilin TKO podocytes. (A-D and I-K) High-magnification images acquired by spinning disk confocal microscopy of podocytes transfected with the indicated pairs of fluorescent fusion proteins. Arrowheads highlight examples of colocalization. Scale bars: $2.5 \mu \mathrm{m}$. (E) Double immunofluorescence labeling demonstrating the increased number of endocytic clathrin-coated intermediates AP2 (alpha-adaptin subunit), and the accumulation of Arp2/3 (p34 subunit) at such intermediates, in pod-Dnm-DKO and Synj1 KO podocytes. Scale bars: $10 \mu \mathrm{m}$. ( $\mathbf{F}$ and $\mathbf{G}$ ) Quantification of the abundance of Arp2/3 and AP2 puncta ( $n=5$ experiments). ${ }^{*} P<0.05$ vs. control. (H) Network of proteins found at endocytic clathrin-coated pits whose loss or functional impairment results in nephrotic syndrome in mice and/or humans. Proteins indicated in red were linked to genetic forms of nephrotic syndrome and focal segmental glomerulosclerosis in humans. Direct interactions are indicated by arrows.

which in neurons supports neurotransmission, is used in podocytes to support formation and maintenance of the glomerular filtration barrier. Akin to what has been proposed for dynamin (5, 11 ), the action of synaptojanin at podocyte foot processes may be due primarily to an effect on actin, because the substrates of the phosphatase activity of Synj1 (namely, PIP2 and PIP3) are critical and potent regulators of actin nucleation $(22,34)$. Likewise, endophilin could have a role in actin function via its property to recruit dynamin, synaptojanin $(19,23)$, and actin-regulatory proteins $(6,35)$. However, it is quite possible that the effects produced by perturbing dynamin, synaptojanin, and endophilin function in podocytes may be mediated primarily by the endocytic roles of these proteins. An even more attractive possibility is that the 2 actions may be tightly interrelated, because actin has been strongly implicated in dynamics of late-stage clathrin-coated pits in a variety of model cell types $(6,36,37)$.

The localization and dynamics of fluorescently tagged Dnm2 (Dnm2-GFP), Synj1-170 (GFP-Synj1), endophilin 2 (Endo2-GFP), and actin in cultured podocytes were examined by spinning disc confocal microscopy. As previously shown in fibroblasts $(28,37$, 38), dynamin selectively colocalized with clathrin at late-stage clathrin-coated pits, just prior to the disappearance of the clathrin signal due to membrane fission and movement of the vesicle away from the plane of imaging concomitant with uncoating (Supplemental Figure 6, A and B, Figure 5C, and Supplemental Video 1). Likewise, as in fibroblasts (28), Synj1-170 was observed at all clathrin-coated pits regardless of maturation state (Supplemental Figure 6, D-F, and Supplemental Video 2), whereas endophilin appeared along with dynamin just before fission (Synj1-170 is not dependent on endophilin for its recruitment, but contains the endophilin binding sites, consistent with a functional interaction of the 2 proteins at late-stage pits; refs. 28,39 ).

As shown in Figure 5A and Supplemental Figure 7A, robust colocalization was observed between Dnm2-GFP-positive spots and spots positive for the mCherry-tagged calponin homology domain of utrophin (mCh-Utr; a probe for F-actin in living cells; ref. 40), demonstrating a transient accumulation of actin, in parallel with dynamin, at the neck of endocytic clathrin pits prior to their fission, as described previously (37). mCh-Utr spots also overlapped with a subset of GFP-Synj1 spots (Figure 5B), representative of late-stage pits (Supplemental Figure 7B), and completely overlapped with Endo2-GFP spots, which also colocalized with mRFPtagged clathrin (mRFP-CLC; Figure 5, C and D, and Supplemen- tal Figure 7, C and D). Thus, while the obligatory recruitment of actin at late-stage endocytic clathrin-coated pits and a cooperative role of dynamin and actin in fission is a matter of debate (38, $41,42)$, a link between actin and clathrin-mediated endocytosis clearly existed in podocytes (Supplemental Video 3). Importantly, Dnm2-GFP, GFP-Synj1, and Endo2-GFP colocalized with F-actin at clathrin-coated pits, not on actin stress fibers (Figure 5, A-C), emphasizing the link between these 3 endocytic proteins and the endocytic function of actin.

The relationship between endocytic clathrin-coated pits and actin in podocytes was further demonstrated by the massive accumulation of Arp2/3 at arrested clathrin-coated pits of cultured podocytes from pod-Dnm-DKO mice (Figure 5, E-G) that were also positive for endophilin (Supplemental Figure 6G), as shown previously in fibroblasts (6). A less pronounced but significant accumulation of Arp2/3 was observed at endocytic clathrin spots of Synj1 KO podocytes (Figure 5, E-G), possibly reflecting a consequence of elevated PIP2 levels at these sites, as previously shown after perturbation of Synj1 function at synapses (21).

Dynamin, synaptojanin, and endophilin interactors play critical functions in podocyte foot processes. In light of the effect that the lack of dynamin, synaptojanin, and/or endophilin has on the glomerular filtration barrier, it is quite striking that other proteins that interacted with dynamin, synaptojanin, and endophilin (Figure $5 \mathrm{H}$ ), when lost, also result in proteinuria (43-45), although their function at foot processes was not attributed to an endocytic function. One such protein is myo$\sin 1 \mathrm{E}(\mathrm{Myo} 1 \mathrm{E})$, a monomeric myosin that binds both dynamin and synaptojanin via a COOH-terminal SH3 domain (45), and whose absence in mice and mutations in humans result in nephrotic syndrome (44-46). When Myo1E was expressed along with either mRFP-CLC or mCh-Utr in podocytes, it accumulated at late-stage clathrin-coated pits, where it colocalized with the pool of F-actin present at these sites (Figure 5, I and J, Supplemental Figure 7, E and F, and Supplemental Video 4). CD2AP, whose functional impairment also results in nephrotic syndrome in both mice and humans $(43,47)$, is a major binding partner of endophilin $(48,49)$ and interacts (like its homolog CIN85) with dynamin and synaptojanin $(50,51)$ both directly and indirectly through endophilin. Accordingly, GFP-tagged CD2AP (CD2AP-GFP), when expressed in podocytes, was localized at late-stage clathrin-coated pits along with endophilin (Figure 5K and Supplemental Figure 7G). Nephrotic syndrome is also produced by loss of Nck, an endocytic adaptor that binds to both dynamin and synaptojanin $(52,53)$. Finally, it has been recently shown that the mouse $\mathrm{KO}$ of $P I 3 K C 2 \alpha$, the gene encoding a class II PI3K that binds clathrin and participates in clathrin-mediated endocytosis (28), develops glomerulonephropathy with proteinuria and renal failure (54).

Endocytic defect in pod-Dnm-DKO and Synj1 KO podocytes. To determine whether the protein network discussed above plays a role in mediating the internalization of key podocyte cargo proteins, we performed internalization assays in isolated podocytes of 2 mutant genotypes, Dnm1;Dnm2 DKO and Synj1 KO. We focused on nephrin as the cargo protein because endocytosis was previously shown to play a role in nephrin regulation (55). Nephrin is an integral membrane protein that participates in the formation of the slit diaphragm that connects adjacent foot processes (56). Podocytes were transfected with a protein chimera comprising the transmembrane and cytoplasmic domain of nephrin and the 
A
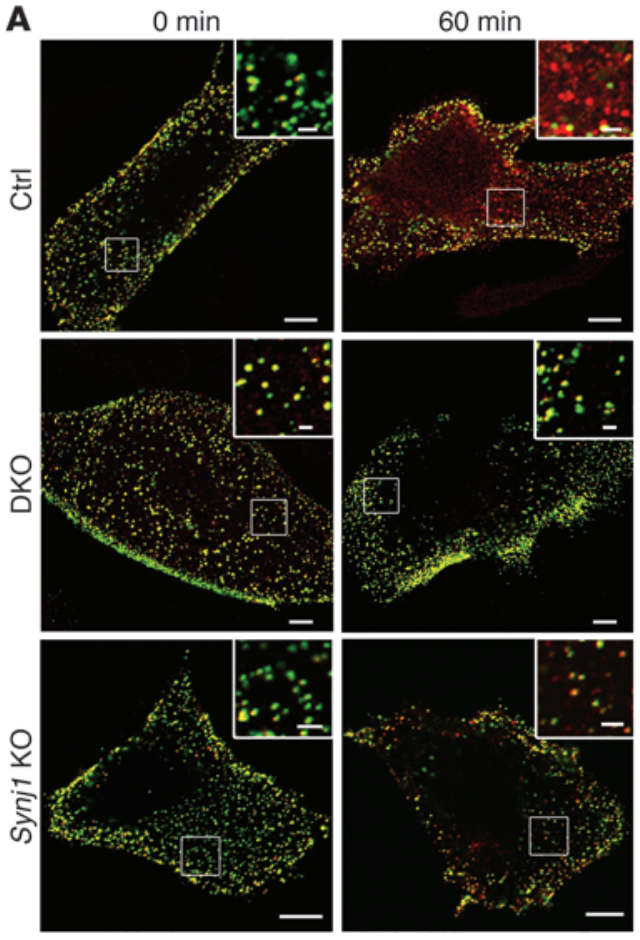

\section{Figure 6}

Nephrin endocytosis is reduced in pod-Dnm-DKO and Synj1 KO podocytes. (A) Internalized (red) versus cell surface (green) CD8-nephrin chimera in control, pod-Dnm-DKO, and Synj1 KO podocytes. Scale bars: $10 \mu \mathrm{m} ; 2 \mu \mathrm{m}$ (insets). (B) Quantification of data in A $\left(n=3 ; 20\right.$ cells evaluated per experiment). ${ }^{*} P<0.01$, pod-Dnm-DKO and Synj1 KO vs. control.

extracellular domain of CD8. Using an anti-CD8 antibody-based internalization assay, we found that the internalization of this probe was significantly delayed in pod-Dnm-DKO and Synj1 KO podocytes (Figure 6, A and B).

\section{Discussion}

Our present findings support an important role of dynamin in the maintenance of the permeability barrier of the kidney (5). We further demonstrated that synaptojanin and endophilin, which are functional partners of dynamin in synaptic vesicle endocytosis at neuronal synapses, were critically implicated in the development of the permeability barrier of the kidney. Most likely, dynamin is also essential for the development of this barrier, but the early embryonic lethality of Dnm2 KO mice did not allow us to explore this possibility. In the genetic model used by us, the expression of Dnm1 and Dnm2 was suppressed after podocyte foot processes have already developed. In contrast, the Synj1 KO and endophilin TKO mice used for our investigations were constitutive KOs. Interestingly, these mice, although greatly neurologically impaired, were macroscopically normal at birth, which indicates that these 2 proteins are critically required only for the development of selected cell types.

Dynamin, synaptojanin, and endophilin have well-established endocytic functions. Endocytosis may help control the steadystate level and turnover of cell surface molecules, which, via actions on signaling pathways, may affect foot process structure. An effect mediated by defective endocytosis, however, does not rule out additional direct effects on actin. All these proteins have been linked to actin function and endocytosis, which are tightly interconnected with actin (Figure 7 and refs. 6, $13,37,38,42)$.

Interestingly, even at synapses, disruption of the function of dynamin, synaptojanin, and endophilin, as well as that of other endocytic proteins, was shown to not only impair endocytosis, but also affect the architecture of nerve terminals, which prompted Dickman et al. to suggest that "endocytosis plays a critical role in sculpting the structure of synapses, perhaps through the endocytosis of unknown regulatory signals that organize morphogenesis at synaptic terminals" (57). In conclusion, our study provides a striking demonstration of how a similar and evolutionary conserved endocytosis machinery, functionally linked to the actin cytoskeleton, has been used in different cellular contexts to control highly specialized physiological process, such as synaptic vesicle recycling and the formation of the glomerular filtration barrier.

\section{Methods}

Antibodies and plasmids. Mouse anti-human CD8 (BD Biosciences - Pharmingen); rabbit anti-WT1, mouse anti-clathrin heavy chain, and rabbit anti-endophilin 3 (Santa Cruz); rabbit anti-p34 Arc/ARPC (Millipore); mouse anti-actin (Sungene Biotech); guinea pig antinephrin (Fitzgerald); rabbit anti-Dnm1 (Epitomics); mouse anti- $\alpha$-adaptin (AP6) (Abcam); Alexa Fluor 488 goat anti-rabbit IgG (Molecular Probes); and Alexa Fluor 594 goat anti-mouse IgG (Molecular Probes) antibodies were obtained commercially. Rabbit anti-Dnm2, rabbit anti-Synj1, rabbit anti-endophilin 1, and rabbit anti-endophilin 2 antibodies were previously described $(6,17)$. Rabbit polyclonal anti-nephrin antibody was provided by Y. Harita (University of Tokyo, Tokyo, Japan; ref. 58). Mammalian expression vectors encoding GFP-Flag-human Synj1-170, mRFP-CLC, and Endo2-GFP were previously reported (28). Dnm2-GFP was provided by M. McNiven (Mayo Clinic, Rochester, Minnesota, USA; ref. 59). mCh-Utr was provided by W. Bement (University of Wisconsin, Madison, Wisconsin, USA; ref. 40). CD8-rat nephrin-Flag construct was provided by Y. Harita (University of Tokyo, Tokyo, Japan; ref. 58). GFP-Myo1E was a gift from M. Mooseker (Yale University, New Haven, Connecticut, USA; ref. 60). CD2AP-GFP was a gift from A. Shaw (Washington University, St. Louis, Missouri, USA; ref. 61).

Generation of mice. $D n m 1^{f / f l}$ and $D n m 2^{f / / f l}$ mice (6) were mated with podCre mice, obtained as previously described (25), to generate the pod-DnmDKO mice with selective glomerular podocyte-specific KO of Dnm1 and Dnm2. Constitutive Synj1 KO (17) and endophilin TKO mice (20) were previously described. Genotyping was performed by PCR using previously described protocols $(6,17,25)$.

Biochemical measurements: plasma creatinine, urine albumin, and urine creatinine. Urine samples were collected from P1-P4 Synj1 KO, P0 endophilin TKO, and littermate control mice and from pod-Dnm-DKO and littermate control mice at various ages. Albuminuria was qualitatively assessed by SDS-PAGE followed by Coomassie Blue staining. Quantitatively, urine albumin was measured using Albumin ELISA Quantitation kit, according to the manufacturer's protocol, in duplicates for 

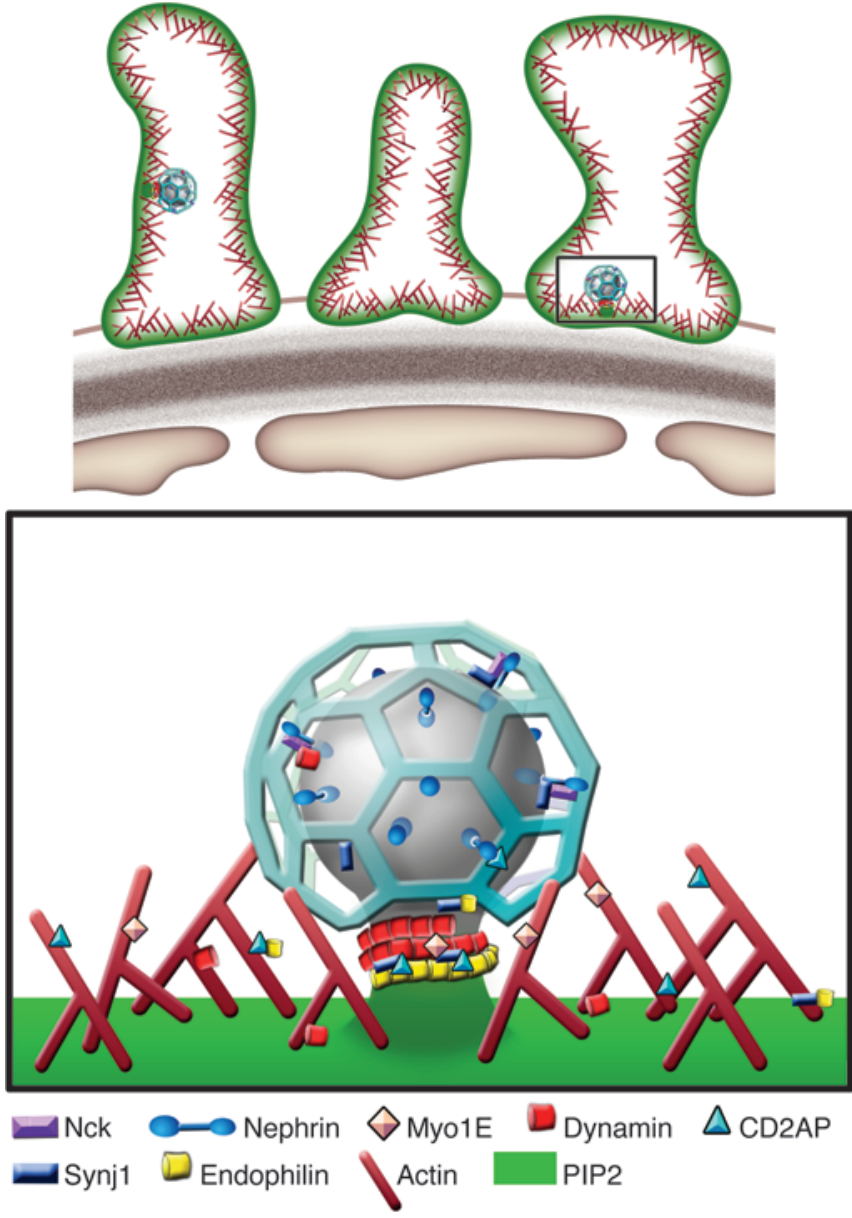

Figure 7

Synaptojanin, dynamin, and endophilin within podocyte foot processes. All the actin-associated proteins shown have interconnected roles in endocytosis, actin regulation, and signaling, and their genetic disruption results in nephrotic syndrome.

each sample (Bethyl Laboratories Inc.). Urine and plasma creatinine were measured in duplicate for each sample by the core facility of the George O'Brien Kidney Center at Yale University.

Cell culture. For Synj1 KO, endophilin TKO, and littermate control mice, whole kidneys were isolated, minced with a razor, and digested with collagenase $(5 \mathrm{mg} / \mathrm{ml})$ containing DNase $(0.2 \mathrm{mg} / \mathrm{ml})$ for 1 hour at $37^{\circ} \mathrm{C}$. The digested kidneys were filtered through a $100-\mu \mathrm{m}$ (Falcon) cell strainer, plated on type 1 collagen-coated dishes, and cultured at $37^{\circ} \mathrm{C}$ in RPMI 1640 medium supplemented with $10 \% \mathrm{FBS}, 100 \mathrm{U} / \mathrm{ml}$ penicillin/streptomycin, $10 \mathrm{mM}$ HEPES, $1 \mathrm{mM}$ sodium bicarbonate, and $1 \mathrm{mM}$ sodium pyruvate. Subculture of primary podocytes was performed from the isolated kidney cells described above by trypsinization with $0.25 \%$ trypsin/ EDTA (Invitrogen) at culture day 3 , followed by sieving through a $40-\mu \mathrm{m}$ cell strainer (Falcon) to isolate an enriched podocyte population as previously described (62). For pod-Dnm-DKO mice, glomeruli were isolated by Dynabeads (Invitrogen) perfusion followed by podocyte isolation as previously described (24). Transfections were performed by electroporation using the Amaxa nucleofactor protocol for podocytes (Lonza).

Immunoblotting. Cells or kidney homogenates were lysed with ice-cold buffer (for Dnm1, Dnm2, or Synj1, $20 \mathrm{mM}$ Tris [pH 7.5], $150 \mathrm{mM} \mathrm{NaCl}$, $1 \%$ Nonidet P-40, $5 \mathrm{mM}$ EDTA, $1 \mathrm{mM} \mathrm{Na} \mathrm{VO}_{4}$, and $50 \mathrm{mM} \mathrm{NaF}$; or $10 \mathrm{mM}$
HEPES-OH [pH 7.4], $1 \mathrm{mM}$ EDTA, $0.32 \mathrm{M}$ sucrose, and 1\% TritonX-100; for endophilin, $10 \mathrm{mM}$ HEPES, $320 \mathrm{mM}$ sucrose, $1 \mathrm{mM}$ EDTA, and 150 $\mathrm{mM} \mathrm{NaCl}$ with protease inhibitor cocktail [Roche Diagnostics]), and protein concentrations were quantified with the Bio-Rad Protein Assay (BioRad). Proteins were separated by SDS-PAGE under reducing conditions, transferred to PVDF membrane (Millipore), blocked with 5\% milk in TBST, and incubated with appropriate primary antibodies in this buffer. After 3 washes with PBS, membranes were incubated with horseradish peroxidaseconjugated secondary antibodies, and signals were detected by enhanced chemiluminescence reagents (GE).

Histology, immunobistochemistry, and immunofluorescence staining. Mice were anesthetized by intraperitoneal injection of ketamine and xylazine followed by perfusion fixation with $4 \%$ paraformaldehyde with or without glutaldehyde through the left ventricle. For histology, sections were sent to Yale Pathology Core Tissue Services for H\&E, periodic acid-Schiff, and Masson's trichrome staining as well as electron microscopy.

For immunohistochemistry, kidney cryosections ( $4 \mu \mathrm{m})$ were subjected to antigen retrieval (Retrievagen; BD Biosciences), followed by blocking with 3\% BSA in PBS for 1 hour. Immunostaining was performed with primary antibodies overnight at $4{ }^{\circ} \mathrm{C}$, followed by Alexa Fluor 488 - and/or Alexa Fluor 594-conjugated secondary antibodies, washing, and mounting with Vectashield with DAPI mounting medium (Vector Labs). Cultured cells were fixed with $4 \%$ paraformaldehyde for 10 minutes at room temperature, washed with PBS, then incubated for 5 minutes in $0.1 \%$ Triton X-100 in PBS, followed by blocking with 5\% BSA for 30 minutes. Cells were then incubated with primary antibodies for 1 hour at room temperature, followed by the appropriate secondary antibody, and imaged using a Perkin Elmer Ultraview Vox spinning disc confocal microscope with either $\times 60$ CFI PlanApo VC (NA 1.4) or $\times 100$ CFi PlanApo VC (NA 1.4) objectives or a Zeiss LSM 710 laser scanning confocal microscope using a $\times 63$ Plan Apo (NA 1.4) oil immersion objective. We used NIH Image $\mathrm{J}$ software to analyze raw images and quantitate particles as previously described (28). For measurement of foot processes/glomerular basement membrane, 4 random glomeruli capillary loops in 3 different animals for each condition were chosen, and glomerular basement membrane length was analyzed by Image J software followed by manually counting the number of foot processes for each loop.

Nonradioactive phosphatidylinositol assay. To evaluate PIP2 levels, a nonradioactive phosphatidylinositol assay was used (63). Briefly, 9 pairs of Synj1 KO and wild-type kidneys were snap-frozen in liquid $\mathrm{N}_{2}$, pulverized, and transferred to a glass tube with $1 \mathrm{ml}$ cold chloroform/MeOH/10 N HCl (20:40:1) containing $2 \mathrm{mM} \mathrm{AlCl}_{3}$. Following homogenization, chloroform and water was added at a 1:2 ratio, the samples were centrifuged, and the lower phase was collected and dried under nitrogen. Phospholipids were deacylated with monomethylamine, resuspended in $0.5 \mathrm{ml}$ water, and extracted twice with an equal volume $n$-butanol/petroleum ether/ethyl formate (20:4:1) to remove fatty acids. The deacylated lipids were recovered by addition of 0.5 $\mathrm{ml}$ water, vortexing, centrifugation, and collection of the aqueous phase. The butanol phase was re-extracted with $0.5 \mathrm{ml}$ water. The second aqueous phase was combined with the first, and the pool was dried under vacuum and resuspended in water. The anionic glycerol head groups were then separated via anion exchange using a Dionex ICS-3000 HPLC system.

Live cell imaging and analysis. Live cell imaging were performed as described previously (28). Briefly, GFP-, mRFP-, and/or mCherry- tagged proteins were coexpressed in primary podocytes by electroporation (Amaxa nucleofector kit R). Transfected cells were seeded in glass-bottomed 35-mm dishes (no. 1.5 thickness; MatTek) and imaged 24 hours later. Before imaging, medium was replaced with an imaging buffer (containing $136 \mathrm{mM} \mathrm{NaCl}$, $2.5 \mathrm{mM} \mathrm{KCl}, 2 \mathrm{mM} \mathrm{CaCl}_{2}, 1.3 \mathrm{mM} \mathrm{MgCl}_{2}$, and $10 \mathrm{mM} \mathrm{HEPES} \mathrm{[pH} \mathrm{7.4]).}$ Cells were imaged using a Perkin Elmer spinning confocal microscope 
(Perkin Elmer) as previously described (6). NIH Image J software was used to analyze images, to generate intensity plots of areas of interest, and to quantitate particles. We calculated the lifetimes of fluorescently tagged proteins by manual assessment of fluorescent spots from images acquired during 3 separate experiments (29). Colocalization between 2 proteins was determined by randomly selecting 20 spots in the green channel, followed by manually scoring for colocalization in the red channel. All data were analyzed for statistical significance using Student's $t$ test.

CD8-nephrin chimera endocytosis assay. For internalization studies, the cells expressing CD8-rat nephrin-Flag were washed with cold PBS, and labeled for 1 hour at $4{ }^{\circ} \mathrm{C}$ with mouse anti-CD8 antibody in serum free RPMI. Unbound antibody was removed by 3 washes with cold PBS prior to incubating the cells at $37^{\circ} \mathrm{C}$ in prewarmed complete RPMI. After internalization of bound label for varying time intervals, the cells were washed with PBS, and surface-retained mouse anti-CD8 antibody was labeled at $4^{\circ} \mathrm{C}$ for 40 minutes with Alexa Fluor 488-conjugated anti-mouse antibody in serum-free RPMI medium, followed by extensive washing with cold PBS and fixation with $4 \%$ paraformaldehyde. The cells were then permeabilized with $0.1 \%$ Triton-X 100 and subsequently blocked with 5\% BSA in PBS, and mouse anti-CD8 antibody was detected with Alexa Fluor 594 anti-mouse antibody. The cells were washed 3 times with PBS, followed by mounting with Vectashield (Vector Laboratories). Images were acquired with a Zeiss LSM 710 laser scanning confocal microscope using a $\times 63$ Plan Ao (NA 1.4) oil immersion objective. To quantify CD8-nephrin endocytosis, fluorescence intensities of cell surface-retained nephrin (Alexa Fluor 488 signal originating from the cell surface) and internalized nephrin (Alexa Fluor 594 signal from the cell interior) were measured with NIH Image J software by examining 20 cells per experiment $(n=3)$ from each indicated time point. We defined the endocytosed nephrin by subtracting the Alexa Fluor 488 image from the Alexa Fluor 594 image and normalizing to surfacederived fluorescence intensity ([Alexa Fluor 594 - Alexa Fluor 488]/Alexa Fluor 488). Anti-CD8 antibody staining on untransfected cells was evaluated as a negative control and found to be minimal.
Statistics. All data are represented as mean \pm SEM. In all cases, group differences were assessed by 2-tailed Student's $t$ test. A $P$ value less than 0.05 was considered significant.

Study approval. All procedures were approved by the Yale University IACUC and followed the guidelines of the NIH Guide for the Care and Use of Laboratory Animals.

\section{Acknowledgments}

We thank Lijuan Liu for excellent technical assistance and Yale Pathology Core Tissue Services for histological analysis. This work was supported in part by NIH grants DK083294 and DK093629 and Norman Coplon Satellite Healthcare Grant to S. Ishibe; by NIH grants R37NS036251, DK082700, and DK45735 to P. De Camilli; by NIH grant George O'Brien Kidney Center at Yale P30DK079310 to S. Ishibe and P. De Camilli; by grants from PRIN2008 to O. Cremona and S. Giovedi; by grants from Cariplo, Telethon, and Associazione Italiana Ricerca Cancro to O. Cremona; by fellowships from the European Molecular Biology Organization and Epilepsy Foundation to I. Milosevic; and by NIH MSTP TG T32GM07205 to D.M. Balkin.

Received for publication June 11, 2012, and accepted in revised form October 11, 2012.

Address correspondence to: Shuta Ishibe, P.O. Box 208029, 333 Cedar Street, New Haven, Connecticut 06520-8029, USA. Phone: 203.737.4170; Fax: 203.785.4904; E-mail: shuta.ishibe@yale. edu. Or to: Pietro De Camilli, 295 Congress Avenue, BCMM 236, New Haven, Connecticut 06510, USA. Phone: 203.737.4461; Fax: 203.737.4436; E-mail: pietro.decamilli@yale.edu.

Silvia Giovedi's present address is: Department of Experimental Medicine, University of Genova, Genova, Italy.
1. Kurihara H, Anderson JM, Farquhar MG. Diversity among tight junctions in rat kidney: glomerular slit diaphragms and endothelial junctions express only one isoform of the tight junction protein ZO-1. Proc Natl Acad Sci U S A. 1992; 89(15):7075-7079.

2. Wiggins RC. The spectrum of podocytopathies: a unifying view of glomerular diseases. Kidney Int. 2007;71(12):1205-1214.

3. Shen K, Bargmann CI. The immunoglobulin superfamily protein SYG-1 determines the location of specific synapses in C. elegans. Cell. 2003;112(5):619-630.

4. Mundel P, Heid HW, Mundel TM, Kruger M, Reiser J, Kriz W. Synaptopodin: an actin-associated protein in telencephalic dendrites and renal podocytes. J Cell Biol. 1997;139(1):193-204.

5. Sever S, et al. Proteolytic processing of dynamin by cytoplasmic cathepsin L is a mechanism for proteinuric kidney disease. J Clin Invest. 2007;117(8):2095-2104.

6. Ferguson S, et al. Coordinated actions of actin and BAR proteins upstream of dynamin at endocytic clathrin-coated pits. Dev Cell. 2009;17(6):811-822.

7. Ferguson SM, De Camilli P. Dynamin, a membrane-remodelling GTPase. Nat Rev Mol Cell Biol. 2012;13(2):75-88.

8. Koenig JH, Yamaoka K, Ikeda K. Omega images at the active zone may be endocytotic rather than exocytotic: implications for the vesicle hypothesis of transmitter release. Proc Natl Acad Sci U S A. 1998;95(21):12677-12682.

9. Ferguson SM, et al. A selective activity-dependent requirement for dynamin 1 in synaptic vesicle endocytosis. Science. 2007;316(5824):570-574.

10. Raimondi A, et al. Overlapping role of dynamin isoforms in synaptic vesicle endocytosis. Neuron. 2011;70(6):1100-1114.

11. Gu C, et al. Direct dynamin-actin interactions regulate the actin cytoskeleton. EMBO J. 2010;29(21):3593-3606.

12. Krueger EW, Orth JD, Cao H, McNiven MA. A dynamin-cortactin-Arp $2 / 3$ complex mediates actin reorganization in growth factor-stimulated cells. Mol Biol Cell. 2003;14(3):1085-1096.

13. Schafer DA, Weed SA, Binns D, Karginov AV, Parsons JT, Cooper JA. Dynamin2 and cortactin regulate actin assembly and filament organization. Curr Biol. 2002;12(21):1852-1857.

14. Pavenstadt H, Kriz W, Kretzler M. Cell biology of the glomerular podocyte. Physiol Rev. 2003;83(1):253-307.

15. Kerjaschki D, Farquhar MG. Immunocytochemical localization of the Heymann nephritis antigen (GP330) in glomerular epithelial cells of normal Lewis rats. J Exp Med. 1983;157(2):667-686.

16. Hayashi M, et al. Cell- and stimulus-dependent heterogeneity of synaptic vesicle endocytic recycling mechanisms revealed by studies of dynamin 1-null neurons. Proc Natl Acad Sci U S A. 2008;105(6):2175-2180.

17. Cremona O, et al. Essential role of phosphoinositide metabolism in synaptic vesicle recycling. Cell. 1999;99(2):179-188.

18. McPherson PS, et al. A presynaptic inositol-5-phosphatase. Nature. 1996;379(6563):353-357.

19. Ringstad N, Nemoto Y, De Camilli P. The SH3p4/ Sh3 $8 /$ SH3p 13 protein family: binding partners for synaptojanin and dynamin via a Grb2-like Src homology 3 domain. Proc Natl Acad Sci U S A. 1997;94(16):8569-8574

20. Milosevic I, et al. Recruitment of endophilin to clathrin-coated pit necks is required for efficient vesicle uncoating after fission. Neuron. 2011;72(4):587-601.

21. Gad H, et al. Fission and uncoating of synaptic clathrin-coated vesicles are perturbed by disruption of interactions with the $\mathrm{SH} 3$ domain of endophilin. Neuron. 2000;27(2):301-312.

22. Di Paolo G, De Camilli P. Phosphoinositides in cell regulation and membrane dynamics. Nature. 2006;443(7112):651-657.

23. de Heuvel E, Bell AW, Ramjaun AR, Wong K, Sos$\sin$ WS, McPherson PS. Identification of the major synaptojanin-binding proteins in brain. J Biol Chem. 1997;272(13):8710-8716.

24. $\mathrm{Ma} \mathrm{H}$, et al. Inhibition of podocyte FAK protects against proteinuria and foot process effacement. J Am Soc Nephrol. 2010;21(7):1145-1156.

25. Moeller MJ, Sanden SK, Soofi A, Wiggins RC, Holzman LB. Podocyte-specific expression of cre recombinase in transgenic mice. Genesis. 2003;35(1):39-42.

26. Saheki Y, De Camilli P. Synaptic vesicle endocytosis. Cold Spring Harb Perspect Biol. 2012;4(9).

27. Ramjaun AR, McPherson PS. Tissue-specific alternative splicing generates two synaptojanin isoforms with differential membrane binding properties. J Biol Chem. 1996;271(40):24856-24861.

28. Perera RM, Zoncu R, Lucast L, De Camilli P, Toomre D. Two synaptojanin 1 isoforms are recruited to clathrin-coated pits at different stages. Proc Natl Acad Sci US A. 2006;103(51):19332-19337. 
29. Schuske KR, et al. Endophilin is required for synaptic vesicle endocytosis by localizing synaptojanin. Neuron. 2003;40(4):749-762.

30. Verstreken P, et al. Synaptojanin is recruited by endophilin to promote synaptic vesicle uncoating. Neuron. 2003;40(4):733-748.

31. Dickman DK, Horne JA, Meinertzhagen IA, Schwarz TL. A slowed classical pathway rather than kiss-and-run mediates endocytosis at synapses lacking synaptojanin and endophilin. Cell. 2005;123(3):521-533.

32. Farsad K, Ringstad N, Takei K, Floyd SR, Rose K, De Camilli P. Generation of high curvature membranes mediated by direct endophilin bilayer interactions. J Cell Biol. 2001;155(2):193-200.

33. Gallop JL, et al. Mechanism of endophilin N-BAR domain-mediated membrane curvature. EMBO J. 2006;25(12):2898-2910.

34. Sakisaka T, Itoh T, Miura K, Takenawa T. Phosphatidylinositol 4,5-bisphosphate phosphatase regulates the rearrangement of actin filaments. Mol Cell Biol. 1997;17(7):3841-3849.

35. Otsuki M, Itoh T, Takenawa T. Neural WiskottAldrich syndrome protein is recruited to rafts and associates with endophilin A in response to epidermal growth factor. J Biol Chem. 2003;278(8):6461-6469.

36. Taylor MJ, Lampe M, Merrifield CJ. A feedback loop between dynamin and actin recruitment during clathrin-mediated endocytosis. PLOS Biol. 2012;10(4):e1001302.

37. Taylor MJ, Perrais D, Merrifield CJ. A high precision survey of the molecular dynamics of mammalian clathrin-mediated endocytosis. PLoS Biol. 2011;9(3):e1000604.

38. Boulant S, Kural C, Zeeh JC, Ubelmann F, Kirchhausen T. Actin dynamics counteract membrane tension during clathrin-mediated endocytosis. Nat Cell Biol. 2011;13(9):1124-1131.

39. Haffner C, Di Paolo G, Rosenthal JA, de Camilli P. Direct interaction of the $170 \mathrm{kDa}$ isoform of synaptojanin 1 with clathrin and with the clathrin adaptor AP-2. Curr Biol. 2000;10(8):471-474.

40. Burkel BM, von Dassow G, Bement WM. Versatile fluorescent probes for actin filaments based on the actin-binding domain of utrophin. Cell Motil Cytoskeleton. 2007;64(11):822-832.

41. Sankaranarayanan S, Atluri PP, Ryan TA. Actin has a molecular scaffolding, not propulsive, role in presynaptic function. Nat Neurosci. 2003;6(2):127-135.

42. Yarar D, Waterman-Storer CM, Schmid SL. A dynamic actin cytoskeleton functions at multiple stages of clathrin-mediated endocytosis. Mol Biol Cell. 2005;16(2):964-975.

43. Kim JM, et al. CD2-associated protein haploinsufficiency is linked to glomerular disease susceptibility. Science. 2003;300(5623):1298-1300.

44. Mele C, et al. MYO1E mutations and childhood familial focal segmental glomerulosclerosis. NEngl JMed. 2011;365(4):295-306.

45. Krendel M, et al. Disruption of Myosin 1e promotes podocyte injury. J Am Soc Nephrol. 2009;20(1):86-94.

46. Sanna-Cherchi S, et al. Exome sequencing identified MYO1E and NEIL1 as candidate genes for human autosomal recessive steroid-resistant nephrotic syndrome. Kidney Int. 2011;80(4):389-396.

47. Shih NY, et al. Congenital nephrotic syndrome in mice lacking CD2-associated protein. Science. 1999;286(5438):312-315.

48. Petrelli A, Gilestro GF, Lanzardo S, Comoglio PM, Migone N, Giordano S. The endophilin-CIN85-Cbl complex mediates ligand-dependent downregulation of c-Met. Nature. 2002;416(6877):187-190.

49. Soubeyran P, Kowanetz K, Szymkiewicz I, Langdon WY, Dikic I. Cbl-CIN85-endophilin complex mediates ligand-induced downregulation of EGF receptors. Nature. 2002;416(6877):183-187.

50. Kowanetz K, et al. CIN85 associates with multiple effectors controlling intracellular trafficking of epidermal growth factor receptors. Mol Biol Cell. 2004;15(7):3155-3166.

51 . Brett TJ, Traub LM, Fremont DH. Accessory protein recruitment motifs in clathrin-mediated endocytosis. Structure. 2002;10(6):797-809.

52. Verma R, Kovari I, Soofi A, Nihalani D, Patrie K, Holzman LB. Nephrin ectodomain engagement results in Src kinase activation, nephrin phosphor- ylation, Nck recruitment, and actin polymerization. J Clin Invest. 2006;116(5):1346-1359.

53. Jones $\mathrm{N}$, et al. Nck adaptor proteins link nephrin to the actin cytoskeleton of kidney podocytes. Nature. 2006;440(7085):818-823.

54. Harris DP, et al. Requirement for class II phosphoinositide 3-kinase C2alpha in maintenance of glomerular structure and function. Mol Cell Biol. 2011;31(1):63-80.

55. Quack I, et al. beta-Arrestin2 mediates nephrin endocytosis and impairs slit diaphragm integrity. Proc Natl Acad Sci US A. 2006;103(38):14110-14115.

56. Kestila M, et al. Positionally cloned gene for a novel glomerular protein-nephrin-is mutated in congenital nephrotic syndrome. Mol Cell. 1998;1(4):575-582.

57. Dickman DK, Lu Z, Meinertzhagen IA, Schwarz TL. Altered synaptic development and active zone spacing in endocytosis mutants. Curr Biol. 2006;16(6):591-598

58. Harita Y, et al. Phosphorylation of Nephrin Triggers $\mathrm{Ca} 2+$ Signaling by Recruitment and Activation of Phospholipase C-\{gamma\}1. J Biol Chem. 2009;284(13):8951-8962.

59. Orth JD, Krueger EW, Cao H, McNiven MA. The large GTPase dynamin regulates actin comet formation and movement in living cells. Proc Natl Acad SciUS A. 2002;99(1):167-172.

60. Krendel M, Osterweil EK, Mooseker MS. Myo$\sin 1 \mathrm{E}$ interacts with synaptojanin-1 and dynamin and is involved in endocytosis. FEBS Lett. 2007;581(4):644-650.

61. Welsch T, et al. Association of CD2AP with dynamic actin on vesicles in podocytes. Am J Physiol Renal Physiol. 2005;289(5):F1134-F1143.

62. Katsuya K, Yaoita E, Yoshida Y, Yamamoto Y, Yamamoto T. An improved method for primary culture of rat podocytes. Kidney Int. 2006;69(11):2101-2106.

63. Nasuhoglu C, et al. Nonradioactive analysis of phosphatidylinositides and other anionic phospholipids by anion-exchange high-performance liquid chromatography with suppressed conductivity detection. Anal Biochem. 2002;301(2):243-254. 